\title{
Increasing oil recovery by utilization of AICV: a 3D multiphase simulation study of a heavy oil reservoir with an underlying water aquifer
}

\author{
T. E. Haugen ${ }^{1}$, A. B. Elverhøy ${ }^{1}$, V. Mathiesen ${ }^{2} \&$ B. M. Halvorsen \\ ${ }^{1}$ Institute of Process, Energy and Environmental Technology, \\ Telemark University College, Norway \\ ${ }^{2}$ InflowControl AS, Norway
}

\begin{abstract}
Oil companies are focusing on the development of new technology to improve the recovery of oil in horizontal wells. Increasing oil recovery represents great financial gains which is important when production facilities are situated in costly locations where access is highly limited, such as offshore environments. One of the main challenges for oil production is when much of the oil in the reservoir has been produced; a breakthrough of either gas, water or both may occur. Breakthroughs lead to increased costs relating to the separation of fluids and operational costs in general. It may also result in the early shut down of the oil field, even though oil is still present. To reduce these problems, Inflow Control Devices (ICDs) is being heavily researched. The Norwegian company InflowControl has patented a new valve named the Autonomous Inflow Control Valve (AICV), which has a promising potential to increase oil recovery factors. Traditional ICDs will only delay the time of breakthrough, while the newly developed AICV will also close the valves, which are producing unwanted fluids. As stated by its name, it works autonomously which means that is it not controlled by any electric or hydraulic form of communication. Using ANSYS FLUENT 13.0, a horizontal well with four AICVs in a reservoir with an underlying aquifer was simulated in 3D. Special attention was given to production rates, breakthrough time and water migration within the reservoir and well. Different pressure resistance was set for each of the four valves to induce a realistic heel-toe effect, which appears by the varying production rates along the well ranging from $20-28 \mathrm{~m}^{3} / \mathrm{d}$. The accumulated oil production when the last
\end{abstract}


valve experienced water breakthrough was approximately $2300 \mathrm{~m}$, which occurred after 22 days of production. Simulation of packers, installed in the annulus between the valves, appear to stop water migration in the annulus in a realistic manner.

Keywords: 3D, AICV, CFD, IOR, heavy oil, heel-toe effect, packers, water breakthrough.

\section{Introduction}

Many horizontally drilled oil fields have underlying water aquifers and overlying gas pockets, relative to the oil reservoir. During oil production, a breakthrough of water or gas may occur at the well; often these breakthroughs are caused by a phenomenon known as the heel-toe effect. At the point of a breakthrough of either phase, the production of oil will be significantly reduced and the need for large separation facilities on the receiving end of the well becomes necessary. Breakthroughs may occur at any position of the well in a heterogeneous reservoir due to varying permeability and cracks in the bedrock formation. However, for a homogeneous reservoir it is likely to occur at the high production section where the drawdown is the highest, i.e. closest to the heel section of the well. Figure 1 shows an example of a horizontal well experiencing breakthrough of both water and gas.

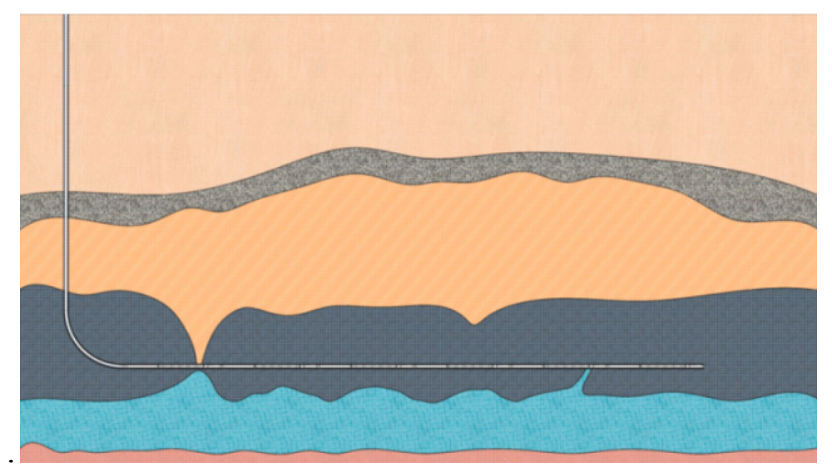

Figure 1: Horizontally drilled well experiencing breakthroughs (Aakre et al. [1]).

Due to the varying drawdown, any underlying water will be drawn up in different amounts along the length of the well, resulting in an inclined water migration profile. This is known as the heel-toe effect. To combat this effect the development of inflow control devices (ICDs) is being researched and implemented on wells. These devices act as pressure drop devices along the well and will thereby give an increase in the time it takes for any breakthrough to occur. Many of the oil industry's biggest companies have developed their own patents of ICDs, each one uniquely designed to delay breakthrough of undesired fluids. This study is focused on a new ICD named the "Autonomous Inflow 
Control Valve" or the AICV. The new patent has several distinct features, which separates it from others, namely that the valve operates autonomously based on the valve's design and is thereby not dependent on any external control communication. Additionally, in contrast to other ICDs it shuts off flow completely when either water or gas reaches the valve and thereby makes sure that only oil is produced from the well. Early estimates by Aakre et al. [1] suggest a potential increase in recovery for heavy oil reservoirs of up to $30 \%$ compared to passive ICDs. This represents a major financial gain in the economy of a producing oil well and supersedes many of the other technologies currently implemented and under development in the field of increasing oil recovery (IOR). To study the potential effects of using the AICV in heavy oil production, a 3D multiphase flow simulation has been performed.

\section{Simulation}

A heavy oil reservoir with an underlying aquifer is simulated in $3 \mathrm{D}$ where oil is produced in a horizontal well through four AICVs. ANSYS FLUENT 13.0 is used for the simulation, while Gambit 2.4 has been used to generate the grid. The domain is divided into four different cell zones; basepipe, annulus, reservoir and the valves. The dimensions of the reservoir are given in Figure 2. In Gambit the grid was made as hexahedral cells for the basepipe, annulus and valves, while the reservoir was made using tetrahedral cells and further converted to polyhedral cells in FLUENT. In total the number of cells was 136768 with varying size from $5.5 \cdot 10^{-6} \mathrm{~m}^{3}$ to $47.1 \mathrm{~m}^{3}$.

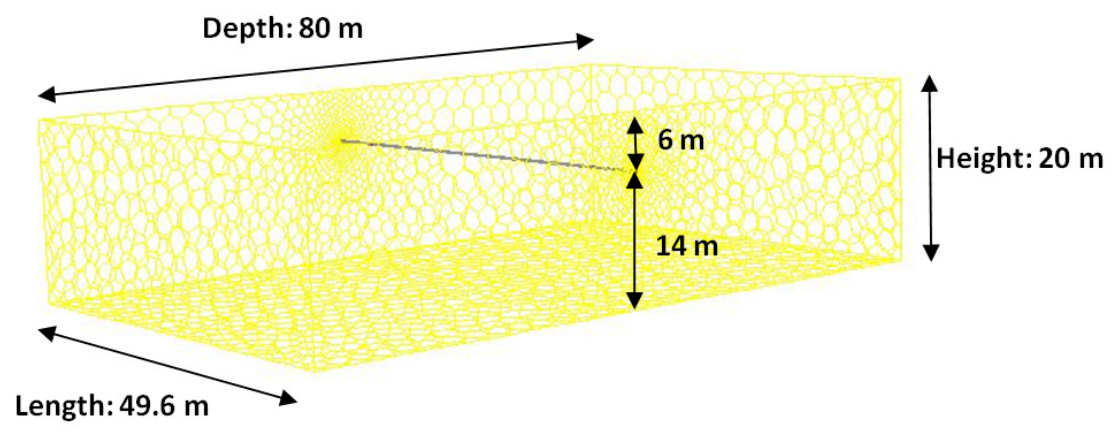

Figure 2: Dimensions of reservoir made with polyhedral grid cells.

A more detailed description of the system is given in Figure 3. The reservoir consists of a $2 \mathrm{~m}$ water layer at the bottom, a $12 \mathrm{~m}$ high oil layer below the basepipe and $6 \mathrm{~m}$ of oil above the pipe. On the basepipe, in between each valve, there are packers installed in the annulus to avoid water migration between valves after water breakthrough has occurred. The boundary conditions are also shown, where pressure inlet is from the bottom of the reservoir and 
pressure outlet is in the positive $\mathrm{x}$-direction of the basepipe. In addition to the pressure boundaries the basepipe and the packers are defined as walls while the valves are defined as interior and the rest are symmetry. The valves are created as $3 \mathrm{D}$ bodies in the annulus with a thickness of $1 \mathrm{~cm}$ which makes the area of inflow larger than that of the real valves. To make sure the flows through the valves are comparable to the AICV, a pressure restriction is included in each valve. The restriction is determined empirically to get the correct flow rates. For simulating the desired heel-toe effect, the lowest restriction is set to the Valve 1 and increased to the left of the reservoir. To be able to close the valves the boundary was manually changed from interior to wall when water breakthrough occurred.

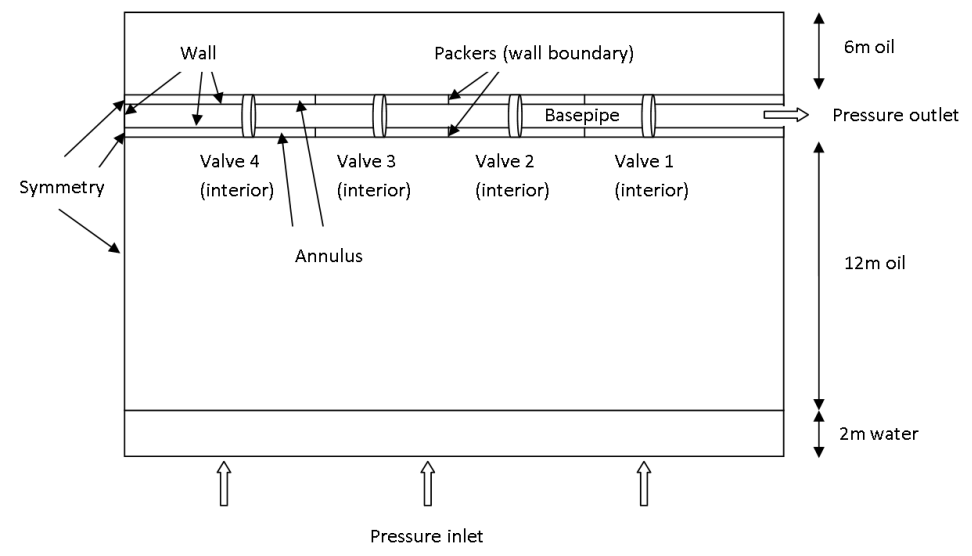

Figure 3: Detailed view of the system with boundary conditions.

The pressure is the driving force in the system and the pressure difference over the reservoir is 5 bar. When also the hydrostatic pressure of the water layer is accounted for the pressure difference will be 6.3 bar in total when the reference pressure outlet is 0 bar. In Table 1 the properties of the fluids and the boundary pressure are given.

Table 1: Boundary conditions and properties of the fluids used in the simulation.

\begin{tabular}{lcc}
\hline \multicolumn{1}{c}{ Properties used in FLUENT } & Value & Unit \\
\hline Reservoir pressure inlet & 6.3 & $\mathrm{bar}$ \\
Reservoir pressure outlet & 0 & $\mathrm{bar}$ \\
Heavy oil viscosity & 100 & $\mathrm{cP}$ \\
Heavy oil density & 970 & $\mathrm{~kg} / \mathrm{m}^{3}$ \\
Water viscosity & 1 & $\mathrm{cP}$ \\
Water density & 998.2 & $\mathrm{~kg} / \mathrm{m}^{3}$ \\
\hline
\end{tabular}


For the simulation the Eulerian multiphase model for immiscible fluid and the implicit volume fraction scheme were used. To solve the pressure-velocity equations the phase coupled SIMPLE algorithm was used. Table 2 shows the selected discretization methods. The initial time step was 1 second and it was gradually increased to 3600 seconds during the simulation.

Table 2: $\quad$ The discretization methods used in the simulation.

\begin{tabular}{ll}
\hline \multicolumn{1}{c}{ Physical property } & \multicolumn{1}{c}{ Scheme } \\
\hline Gradient & Least square cell based \\
Momentum & Second order upwind \\
Volume fraction & Modified HRIC \\
Transient & Second order implicit \\
\hline
\end{tabular}

\section{Results}

Figure 4 shows the full length of the well with the four valves producing towards the right side (heel). In addition the drawdown pressures acting in the surrounding area of each valve are presented. At the start of the simulations the drawdown along the well is highest at the heel section and decreases towards the toe section (left) of the reservoir.

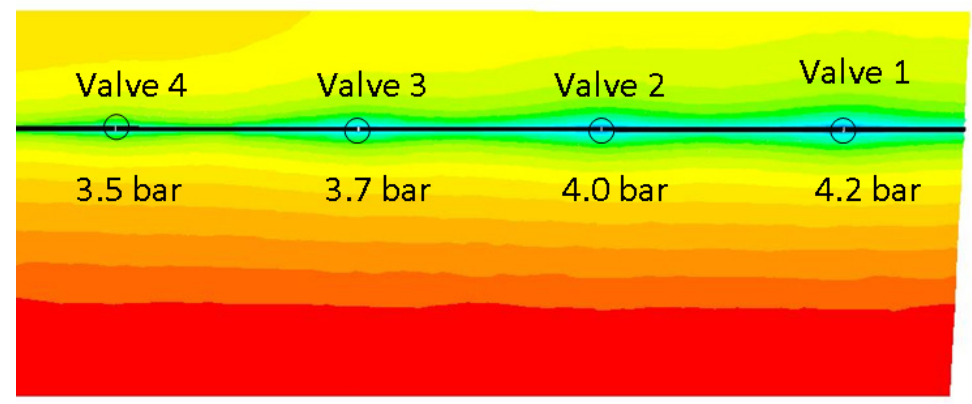

Figure 4: The horizontal well with valves and drawdown pressures.

The result of the varying drawdowns is presented in Figure 5 where the phase contours initially and after 18 days shows the water migration towards the well, with water as the phase appearing under the oil phase. To the right side in the figure, the reservoir condition just before water breakthrough occurs in Valve 1 is shown. Meanwhile the other valves are still producing oil. The gradient of the migrating water indicates a successful simulation of the heel-toe effect. 

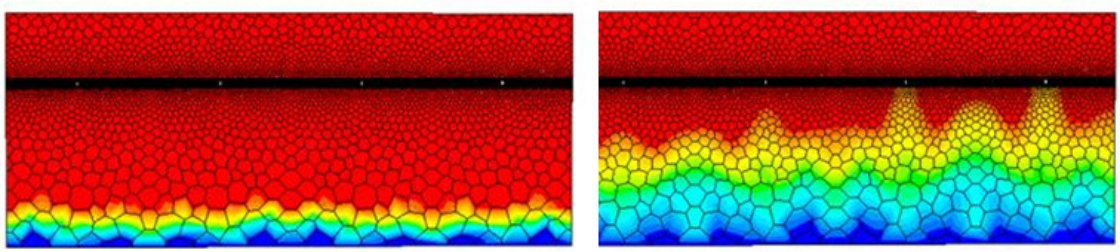

Figure 5: Phases at initial conditions and after 18 days.

During oil production oil will flow into the annulus from the reservoir and migrate to the valve inlet, which allows oil to flow into the basepipe. This migration of oil was observed during the simulations and is displayed in Figure 6 . It is clearly shown by the velocity vectors that the oil migrates towards the valve inside the annulus. As it enters the small valve inlet into the basepipe the velocity increases. Once inside the basepipe the velocity profile is that of a typical pipe flow situation, where the highest velocity is occurring in the center of the pipe and that the velocity at the pipe wall is close to zero. It is also observed by the vectors that the total velocity inside the basepipe increases as more oil is drawn in to the basepipe through the valve.

In Figure 7 the production rates from each valve are presented. The figure shows that each valve increases its flow rate from the initial production of 20-28 $\mathrm{m}^{3} / \mathrm{d}$ to $25-35 \mathrm{~m}^{3} / \mathrm{d}$ at water breakthrough. As one valve closes, there is an increase of the production rate in the nearest valve due to the increased pressure difference occurring in the basepipe. The breakthrough times for each of the valves are determined in, which varies from 18-22 days.

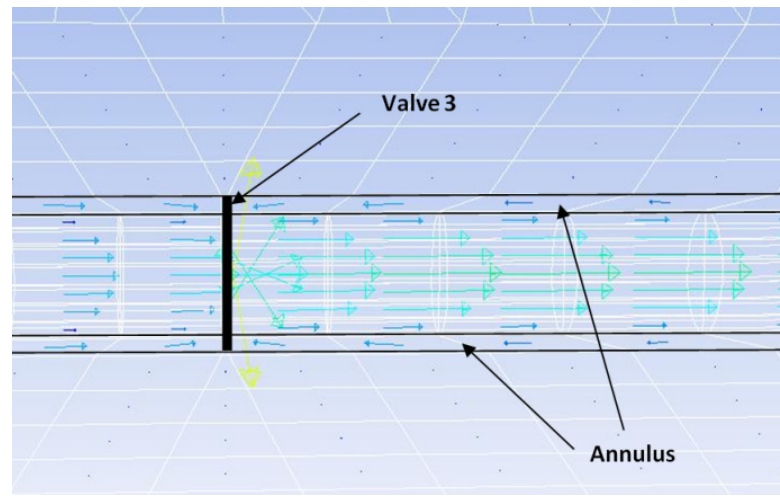

Figure 6: Fluid behavior in the well. 


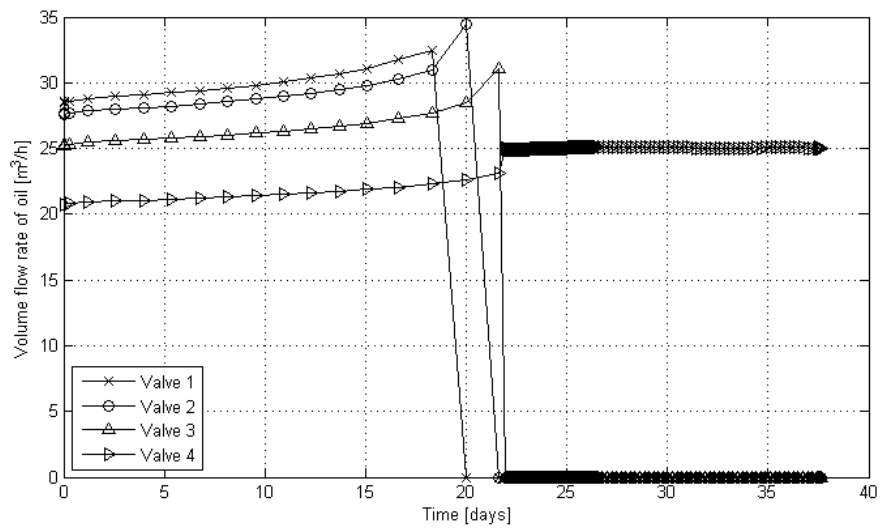

Figure 7: $\quad$ Production rates for each valve.

Once Valve 4 experiences breakthrough the simulations were continued with the valve fully open to investigate water production relative to oil production. In Figure 8 it is observed that the oil production rate remain fairly constant while the water production gradually increases with time. As a result of this the wateroil-ratio (WOR) gradually increases, which is shown in Figure 9.

The accumulated oil production was approximately $2300 \mathrm{~m}^{3}$ when the last valve experienced breakthrough on day 22. For the total simulation the accumulated oil production was $2700 \mathrm{~m}^{3}$ on day 37 , at which point the WOR became so high that continuing production yields very little oil.

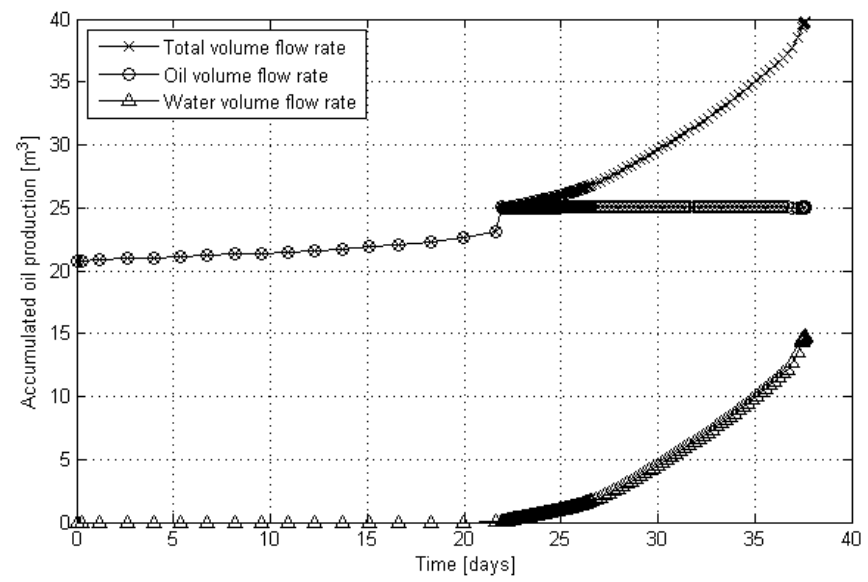

Figure 8: Phase flow rates. 


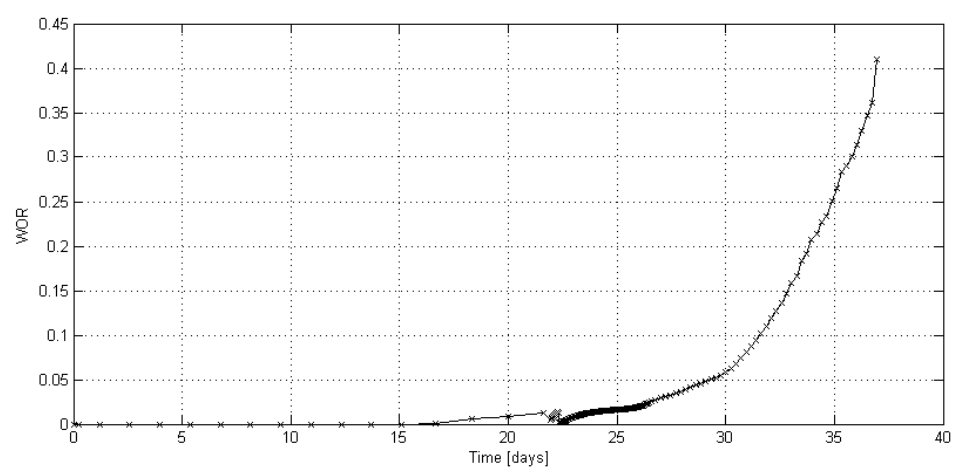

Figure 9: WOR ratio throughout the simulation.

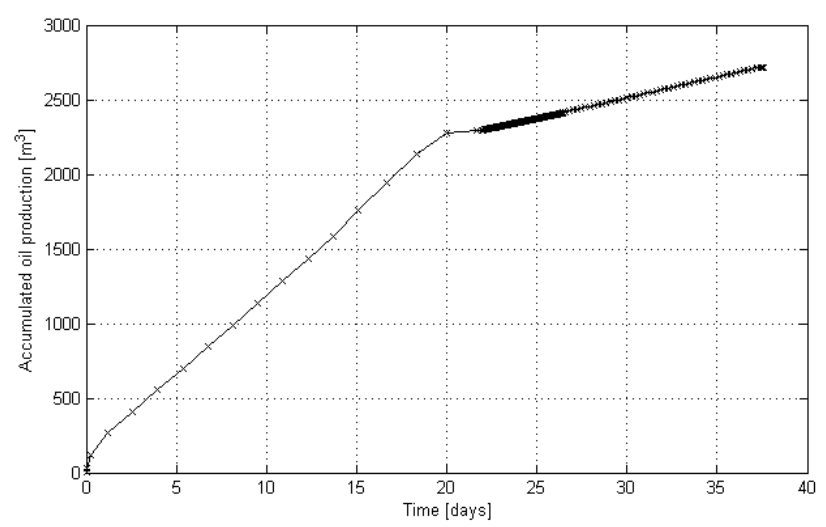

Figure 10: Accumulated oil production.

Water migration is illustrated in Figure 11 at three different points in time. In the figure the oil is not visible and the contours of the water surface approaching the well are shown as a silhouette. At the top picture Valve 2 are already closed while Valve 3 is just closing. Once closed the water in the annulus approaches and accumulates at the packer between Valve 3 and 4, which is illustrated in the picture in the middle. This gives indication that the packers modeled for the simulations works as intended. Water is observed to migrate back into the reservoir at the packers, but does not migrate any further. This is due to the different permeability defined in the reservoir and annulus, where the low permeability in the reservoir restricts the flow. In the bottom picture the water profile after all valves have experienced breakthrough is shown. At this point Valve 4 is still open and producing with a high WOR. It also illustrates how a potential water profile in a heavy oil reservoir would look like with the use of AICV technology. 

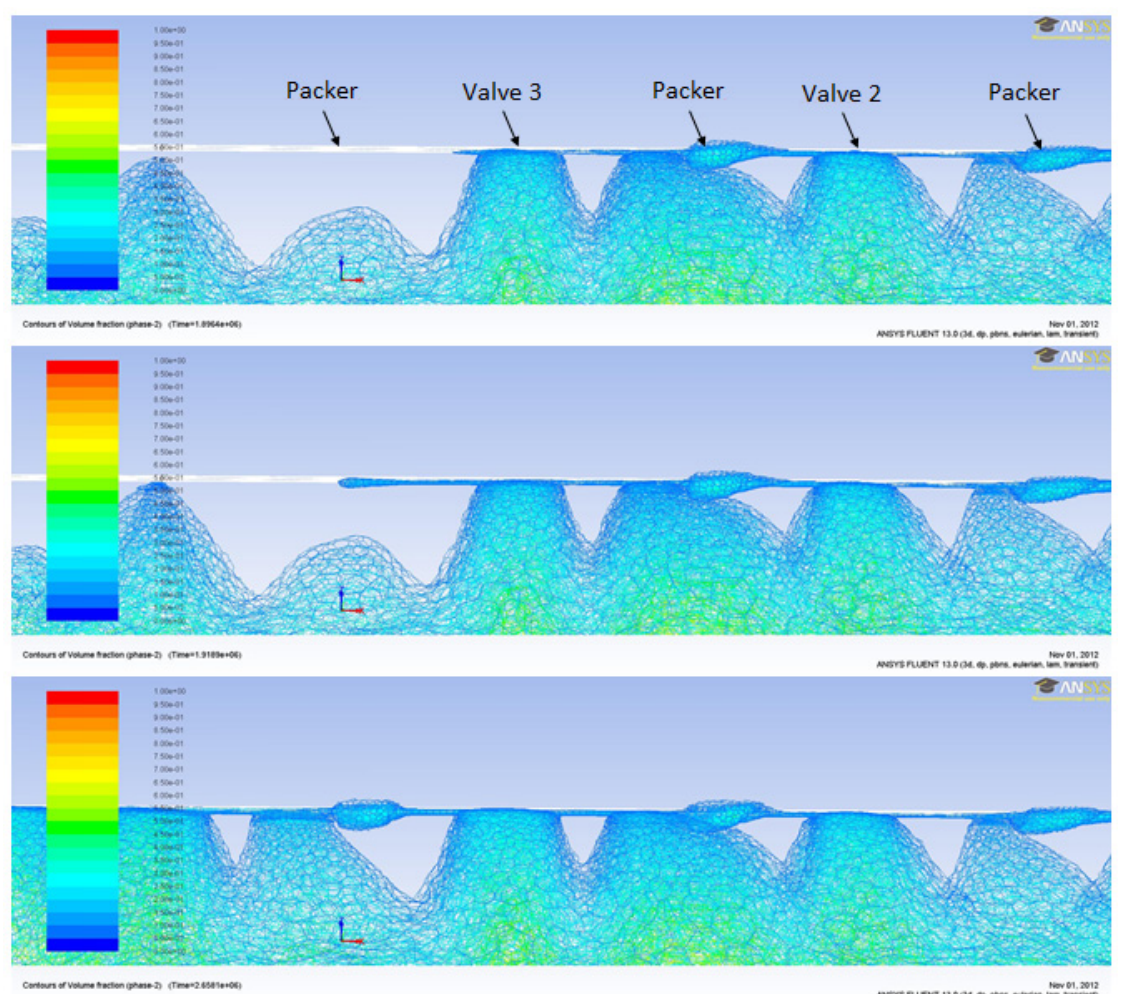

Figure 11: Water migration at three different points in time. Top: 21.9 days, middle: 22.2 days, bottom 30.8 days.

\section{Conclusion}

A 3D simulation of a heavy oil reservoir with an underlying aquifer has been performed in order to investigate the potential effects of implementing AICVs in a horizontal well. ANSYS FLUENT 13.0 was used as the simulation tool with the Eulerian multiphase model for immiscible fluids and the implicit calculation scheme for volume fractions. Special attention was given to oil production rates, water breakthrough times and water migration. The four valves along the well showed initial production rates varying from $20-28 \mathrm{~m}^{3} / \mathrm{d}$. These rates gradually increased during production until water breakthrough occurred at which the valves were closed manually. The breakthrough times were found to vary from 18-22 days. At the closing of the last valve the accumulated oil production was approximately $2300 \mathrm{~m}^{3}$. As for water migration in the reservoir, the induced heel-toe effect was considered successful. When the valves closed water migrated inside the annulus towards the next open valve, but was stopped by the 
1254 Energy Production and Management in the 21st Century, Vol. 2

packers. This is considered a realistic simulation of the packers intended function.

\section{Reference}

[1] Aakre, H. Halvorsen, B. Werswick, B. Mathiesen, V., Smart well with autonomous inflow control valve technology, SPE 164348-MS SPE Middle East Oil and Gas Show and Exhibition held in Manama, Bahrain, 10-13 March 2013. 